\title{
DEFICIÊNCIA DE FERRO E DESENVOLVIMENTO COGNITIVO
}

\section{IRON DEFICIENCY AND COGNITIVE DEVELOPMENT}

\author{
Edna Helena da Silva Machado ${ }^{1}$ \\ Claudio Leone ${ }^{2}$ \\ Sophia Cornbluth Szarfarc ${ }^{3}$
}

Machado EHS; Leone C; Szarfarc SC. Deficiência de ferro e desenvolvimento cognitivo. Rev Bras Cresc e Desenv Hum 2011; 21(2): 368-373.

\section{RESUMO}

É aceito que a deficiência de ferro acarreta conseqüências deletérias para o desenvolvimento cognitivo, no entanto, é difícil quantificar esse papel uma vez que ele é determinado conjuntamente com fatores sócio-econômico-ambientais. Mesmo assim, o controle da anemia diminui uma fração do risco do subdesenvolvimento cognitivo e isso significa um avanço positivo na qualidade de vida. Tendo em mente a ampliação do conceito de saúde para o de preservação da vida com qualidade, as ações dirigidas à atenção à saúde devem ser incorporadas às ações governamentais programáticas mais abrangentes e intersetoriais. Este trabalho tem como objetivo fazer uma reflexão sobre o papel da escola no desenvolvimento global da criança destacando o atendimento das necessidades nutricionais de ferro tendo em vista evitar que a deficiência marcial seja um limitante para a capacitação social do indivíduo.

Palavras-chave: desenvolvimento cognitivo, anemia ferropriva, necessidade nutricional de ferro

1 Coordenadoria Regional de Saúde-Centro Oeste - E-mail: ehe_cap@ig.com.br. Avenida Renato Paes de Barros, 77 - Itaim Bibi. São Paulo-SP

2 Faculdade de Saúde Pública/USP. Departamento de Saúde Materno Infantil. Av. Dr. Arnaldo, 715. São Paulo-SP. Cep: 01246-904 - E-mail: leone.claudio@gmail.com

3 Faculdade de Saúde Pública/USP. Departamento de Nutrição. Av. Dr. Arnaldo, 715. São Paulo-SP. Cep: 01246-904 - E-mail: scfarc@usp.br

Correspondência para: Sophia Cornbluth Szarfarc. E-mail: scfarc@usp.br 


\begin{abstract}
It is universally accepted that anemia determined by iron deficiency leads to deleterious consequences for the cognitive development. However, it is difficult to quantify the role of anemia determined in conjunction with socio-economic-environmental factors. Even so, the control of anemia reduces a fraction of risk for cognitive underdevelopment and it means a positive advance in life quality. Considering the extended concept of health for the preservation of life with quality, the actions directed to health care should be incorporated into more comprehensive and intersectorial government program actions. This work aims to discuss the role of school in the overall development of the child, pointing out the compliance with iron nutritional requirements, aiming to prevent martial deficiency from being a limiting factor for the individual's social capacity.
\end{abstract}

Key words: cognitive development, iron deficiency anemia, iron requirement

\section{INTRODUÇÃO}

A importância social da educação formal, com a escola assumindo o papel de propiciar, estimular e orientar o desenvolvimento global da criança faz o desempenho escolar sobressair como uma área em que é fundamental estabelecer a influência de quaisquer condições que possam levar a uma ruptura do estado dinâmico de equilíbrio do indivíduo, ultrapassando os limites frágeis e imprecisos do binômio: saúde - doença, dentre os quais a deficiência de ferro ocupa um espaço importante.

A emergência de controlar agravos que não encontram resposta no âmbito do setor saúde isoladamente provocou a ampliação do conceito de saúde para o de preservação da vida com qualidade. Portanto, a noção de saúde deve ser abordada como processo dinâmico e continuo e conseqüente das ações do indivíduo e da sociedade ${ }^{1}$.

Esta forma de entender a saúde exige uma nova prática de atenção à mesma como uma forma de resposta social organizada aos problemas, considerando o ser humano como um ser inserido num contexto sócio-cultural. Sendo assim, o modelo de atenção à saúde deve ter como objetivo de evoluir de forma a manter um sistema de vigilância à qualidade de vida ${ }^{1}$.
Para alcançar este objetivo, as ações dirigidas à atenção à saúde devem ser incorporadas às ações governamentais programáticas mais abrangentes e intersetoriais.

A Escola é um espaço adequado para a implantação de política pública que visa a promoção da saúde, e, desenvolver a vigilância dos distúrbios nutricionais deve fazer parte das atividades escolares.

Dentre as principais deficiências nutricionais, a anemia ocupa lugar de destaque. Em escolares é particularmente deletéria, pois crianças anêmicas apresentam sonolência durante as atividades e com isso a sua atenção fica prejudicada levando ao baixo rendimento escolar.

A deficiência de ferro tem sido destacada como o problema nutricional de maior impacto quer em relação ao crescimento, em relação ao desenvolvimento psicossocial como, também, em relação ao desenvolvimento motor e cognitivo.

Crianças anêmicas têm sido consideradas as mais sensíveis a agravos no desenvolvimento cognitivo e neuro-psicomotor em função dos comprometimentos de processos fisiológicos relacionados à produção de hemoglobina e conseqüente transporte de oxigênio ao cérebro, alterando a neuro transmissão e mielinização ${ }^{2}$. 
Devido às dificuldades decorrentes dos inúmeros fatores sociais, ambientais e econômicos que concorrem para o desenvolvimento de uma criança, poucos são os estudos que avaliam a inter-relação entre deficiência de ferro e desenvolvimento cognitivo. No entanto, como refere Grantham-McGregor ${ }^{3}$, a possibilidade que a deficiência de ferro afete o desenvolvimento infantil é um sério problema de saúde pública e traz implicações não somente para o desenvolvimento pessoal, mas também para as nações em desenvolvimento onde a prevalência da anemia é sempre elevada ${ }^{4}$. Comprovar a associação entre anemia e desenvolvimento cognitivo e motor é ainda mais dificultado por ser a deficiência de ferro determinada por muitos fatores sócios econômicos como escolaridade, renda e qualificação profissional.

Sendo assim, os resultados descritos em estudos que visam identificar as relações de anemia com o desenvolvimento cognitivo devem ser vistos com cautela uma vez que se torna muito difícil controlar todas as variáveis de confusão. A maior parte desses estudos foi desenvolvida entre lactentes e, com todas as dificuldades inerentes aos estudos longitudinais. Alguns deles permitiram tecer considerações sobre os efeitos a longo prazo decorrente da anemia nos primeiros dois anos de vida sobre as funções mental, motor, social/emocional e neurofisiológicas 5 .

Uma alimentação deficiente em ferro pode alterar significativamente o desenvolvimento do sistema nervoso central como um resultado de alterações na morfologia, neuroquímica e bioenergética. Dependendo do estagio em que a carência do mineral ocorre, ou quanto mais precocemente essa deficiência nutricional é corrigida, maiores são as oportunidades de reverter os efeitos adversos decorrentes dessa alimentação ${ }^{6}$.

Dentre os poucos estudos nacionais que tratam conjuntamente do desenvolvimento cognitivo e da anemia destaca-se o de Santos et $\mathrm{al}^{7}$ que avaliaram o desenvolvimento de lin- guagem de crianças distribuídas segundo a presença ou não de anemia, com idades entre dois e seis anos de idade, freqüentadoras de creche assistencial de Belo Horizonte, Minas Gerais. Os autores verificaram que crianças anêmicas apresentaram pior desenvolvimento na linguagem com diferenças estatisticamente significantes em relação aos aspectos comunicativos (recepção e emissão de informações) assim como a aspectos cognitivos da linguagem. Como referem os autores, esse resultado não pode ser aceito como sendo de uma relação causa efeito entre anemia e desenvolvimento de linguagem uma vez que as alterações neurocognitivas estão sujeitas a inúmeras variáveis decorrentes da historia pregressa e atual das crianças.

Esse limite para os estudos que pretendem avaliar a influência da deficiência de ferro e desenvolvimento cognitivo já havia sido descrito por Moisés ${ }^{8}$, em 1979, em estudo que avaliava a relação entre esses dois parâmetros. Com uma amostra de 300 estudantes de $1^{\mathrm{a}}$ a $7^{\mathrm{a}}$ série, a pesquisadora avaliou três áreas do desenvolvimento cognitivo consideradas pré-requisitos para a aprendizagem: memória visual, através de Teste de Retenção Visual de Benton, coordenação visomotora, avaliada pelo teste Gestáltico Visomotor de Bender e o vocabulário utilizando o Peabody Picture Vocabulary Test, adaptado para o Brasil.

O rendimento escolar foi avaliado através das notas conferidas aos alunos amostrados pelos professores de português e de matemática. O teste de memória visual foi aparentemente, o único indicador que revelou desempenho mais precário entre o grupo de anêmicos quando comparado ao grupo controle.

A deficiência de ferro se apresenta como um dos fatores limitantes do desenvolvimento cognitivo havendo melhora significante de memória visual após a suplementação com o mineral. Após o controle da deficiência marcial, o grupo de (ex) anêmicos passou a ter uma memória visual similar ao do grupo controle 
não anêmico. As outras áreas avaliadas e o rendimento escolar não mostraram diferenças significativas entre os grupos com e sem deficiência de ferro.

Como destaca Moisés ${ }^{8}$ discutindo os resultados encontrados no seu estudo, o homem sofre a influência de variáveis que interferem no desenvolvimento global e o que é avaliado como desempenho cognitivo é resultado de uma interação bastante complexa entre sua capacidade de desenvolvimento potencial e fatores ambientais. Em relação ao desenvolvimento cognitivo, a influência do ferro é dificilmente perceptível frente a fatores, especialmente sociais, que determinam este desenvolvimento.

Moisés ${ }^{8}$ chama a atenção também para o fato da memória visual ser muito mais independente de fatores ambientais que a coordenação visomotora e o vocabulário. Para este último o desenvolvimento está intimamente relacionado ao estímulo produzido pelo ambiente social que será imitado e eternizado. Com relação à avaliação escolar, ela aponta para a relatividade da avaliação pelos professores dada a interação relação-aluno, as diferenças entre os professores, e pela subjetividade do processo.

Kleiner ${ }^{9}$ destaca que a capacidade da linguagem está presente de modo crucial em todos os aspectos da vida, do pensamento e da interação humanos.. Apesar da criança não precisar do pensamento consciente para desenvolver a linguagem, com a formação de frases corretamente elaboradas, os índices de fracasso escolar atingem cerca de $20 \%$ das crianças escolarizadas em ensino normal.

Mesmo com todos esses multifatores influindo no desenvolvimento cognitivo, certamente a deficiência do ferro é um importante fator limitante desse desenvolvimento por ser elemento fundamental para que os processos oxidativos do metabolismo se processem, e que, se deficiente pode provocar alteração de todo o metabolismo celular com repercus- sões em todos os órgãos e sistemas, inclusive no sistema nervoso central.

O modelo atual das escolas, que não impede o avanço escolar de crianças não preparadas para a progressão formal esperada para cada idade/conteúdo programático, não permite conhecer as relações entre deficiência de ferro e desempenho escolar. Estudos da década de 90, no entanto, mostravam claramente a maior proporção de crianças anêmicas entre repetentes de uma série escolar ${ }^{10}$.

Também foi verificado que à medida que a progressão escolar ocorria, diminuía a prevalência da anemia e a idade média tendia a se aproximar da esperada para o ano escolar. Essa ocorrência certamente é decorrente quer da reprovação, deixando alunos em atraso e/ou abandono da escola o que, por sua vez, permite supor que o abandono era mais freqüente entre os ferro deficientes. Por sua vez, os professores, em geral, possuem um conhecimento superficial a respeito dos transtornos de aprendizagem e possuem uma visão limitada quanto à atuação desses transtornos para o desenvolvimento global da criança ${ }^{11}$. Em um levantamento da prevalência de anemia entre escolares da rede pública de ensino de Santos, Stefanini relatou ter encontrado $27,9 \%$ de anêmicos entre crianças ingressantes, $11,3 \%$ entre as da $5^{\text {a }}$ série e 5,6\% entre aquelas que estavam completando o ensino fundamental $\left(8^{\mathrm{a}}\right.$ série). A proporção de classes escolares entre a $1^{\mathrm{a}}$ e a $8^{\mathrm{a}}$ séries era de 20:1 sendo que em várias escolas as classes eram restritas as primeiras 4 séries $^{12}$.

Há relação entre deficiência de ferro e atraso no desenvolvimento cognitivo e psicomotor de lactentes, pois em crianças desta faixa etária ocorre a maior prevalência de anemia $^{13,14}$.

A possibilidade de a anemia afetar o desenvolvimento infantil é indiscutivelmente, um problema de saúde pública dos mais sérios visto ter implicações que se estendem por toda a vida e afetam não somente os indivíduos mas se 
reflete no desenvolvimento nacional de comunidades onde a prevalência da deficiência de ferro é elevada. Estudos desenvolvidos por Lozoff et $\mathrm{al}^{14}$ ressaltam que crianças anêmicas apresentam escores menores em relação ao desenvolvimento motor e mental. Mesmo após o controle da deficiência marcial as diferenças de desempenho nessas áreas, quando comparadas a crianças que nunca apresentaram ferro deficiência se mantiveram. Igualmente foi observada que a reprovação escolar ocorria entre elas com maior freqüência. Familiares concordavam com os professores a respeito do comportamento ansioso/ depressivo das crianças que também apresentavam déficits de atenção ${ }^{15}$.

Assim sendo, o controle da anemia deve ser alvo da preocupação da saúde visando evitar que as crianças sejam vitimas dessa deficiência nutricional e se transformem em indivíduos com baixa escolaridade e produtividade quando adultos causando perdas de recursos humanos com conseqüências econômicas e sociais para o desenvolvimento da nação.

Horton e Ross ${ }^{16}$ mostram que não pode ser desprezado o custo indireto da deficiência de ferro na infância, a qual tem conseqüências irreversíveis sobre o desenvolvimento cognitivo e sobre a produtividade durante a vida adulta. Eles enfatizam a importância da intervenção para o controle dessa morbidade e concluíram que, tanto a fortificação de alimentos habituais na alimentação da população alvo, como a suplementação medicamentosa, se efe- tivamente implantadas, constituem um investimento pequeno (inferior a $0,3 \%$ do PIB de países em desenvolvimento).

Tem sido grande o número de pesquisadores ligados à saúde atraídos pelas possíveis conseqüências de distúrbios nutricionais sobre o desenvolvimento mental e comportamental do ser humano. A maioria desses estudos que trata os efeitos somáticos da deficiência de ferro, tanto sobre a unidade celular como sobre o organismo como um todo, são precariamente compreendidos. Ainda mais difícil é a análise da influência do ferro sobre aspectos funcionais do sistema nervoso central. Esta dificuldade decorre, basicamente, da impossibilidade de isolar os efeitos da deficiência do mineral de outros fatores ambientais: econômicos, sociais e culturais que constantemente influem sobre o indivíduo e ainda mais sobre o desenvolvimento principalmente na criança. Não se pode, pois considerar que a deficiência nutricional do ferro, seja a única responsável pelos agravos observados no desenvolvimento neuropsicomotor.

Se, por um lado, mudanças sociais são dificilmente conseguidas a curto/médio prazo, o controle da deficiência nutricional do ferro pode ser alcançado entre crianças que são beneficiadas por programas educacionais, seja em creches e escolas com a oferta de alimentos fortificados com o mineral. Embora os problemas sociais não se resolvam na sua integra, esse é um caminho para a introdução da criança na sociedade.

\section{REFERÊNCIAS}

1. Starfield B. Atenção primária. Equilíbrio entre necessidades de saúde, serviços e tecnologia. Brasília: Ministério da Saúde; 2002. 19-70.

2. Granthan-MacGregor S. A review of studies on the effect of iron deficiency on cognitive development in children. J.Nut.2001;131(2S):649-668.

3. Granthan-MacGregor S. Does IronDeficiency Anemia Affect Child 
Development? .Pediatrics. 2003; 112(4):846-54.

4. World Health Organization (WHO). Iron deficiency anemia: assessment prevention and control: a guide for Programme Managers. Geneva: WHO; 2001.

5. Patrício D, Peiriano CA, Marcelo I, Garrido Betsy Lozoff. Iron deficiency anemia in infancy is associated with altered temporal organization of sleep states in childhood. Pediatr Res. 2007;62(6):75-19

6. Beard J. Why Iron Deficiency Is Important in Infant Development. J Nutr.2008;138:2534-36.

7. Santos JL, Rates, SPM, Lemos SMA, Lamounier, J. Anemia em crianças de uma creche pública e as repercussões sobre o desenvolvimento de linguagem. Rev Paul Pediatria. 2009;27:1-9.

8. Moisés MA. Deficiência de ferro e desenvolvimento cognitivo: um estudo experimental em escolares. Pediatria (São Paulo). 1981;3(3):217-25.

9. Kleiner, H. Problemas de aquisição de leitura e escrita: proposta de atendimento com as técnicas psicopedagógicas preventivas para crianças e adolescentes. Anais do II Congresso Internacional de Saúde da Criança e do Adolescente. Rev Bras Crescimento Desenvolvimento Hum. 2011; 21(2): 419.

10. Cesar, AT. O efeito do ácido ascórbico no controle da deficiência de ferro utilizando a estrutura do Programa da Merenda Escolar [dissertação mestrado]. São Paulo: Faculdade de Saúde Pública-; 1990.
11. Machado, AC; Capellini, AS. Visão dos professores do ensino público sobre transtornos de aprendizagem. Anais do II Congresso Internacional de Saúde da Criança e do Adolescente. Rev Bras Crescimento Desenvolvimento Hum. 2011; 21(2): 485

12. Lerner, BR A alimentação e a Anemia Carencial em adolescentes [tese de doutorado]. São Paulo: Faculdade de Saúde Pública-; 1994.

13. Lozoff B, Beard J, Connor J, Barbara F, Georgieff M, Schallert T. Long-lasting neural and behavioral effects of iron deficiency in infancy. Nutr Rev. 2006; 64(5 Pt 2):S34-43.

14. Lozoff B, Jimenez E, Hagen J, Mollen E, Wolf AW. Poorer behavioral and developmental outcome more than 10 years after treatment for iron deficiency in infancy. Pediatrics. 2000; 105(4):E51.

15. Lozoff B, Jimenez E, Smith JB. Double burden of iron deficiency in infancy and low socioeconomic status: a longitudinal analysis of cognitive test scores to age 19 years. Arch Pediatr Adolesc Med. 2006; 160:1108-13.

16. Horton S, Ross J. The economics of iron deficiency. Food Policy. 2003; 28(1): 51-75.

Recebido em: 26/out./2010

Modificado em 06/fev./2011 Aceito em 25/mar./2011 